\title{
Does GP fundholding affect the use of tertiary services in the UK?
}

\author{
Nick Black, Susan Langham
}

Since 1991, general practitioners (GPs) in the UK have been allowed to manage part of the NHS funds allocated for the secondary care of their patients. This has included outpatient consultations, pharmaceuticals, community services, and elective surgery. Despite the growing body of research on the impact of such GP fundholding, ${ }^{1}$ there have been no reports on its effect on the use of tertiary services. This is partly because the purchase of most of the services provided by tertiary centres has remained the responsibility of health authorities. There has, however, been one important exception, that of elective surgery. And if total purchasing (in which GPs control funds for all secondary and tertiary services used by their patients) were to be widely adopted, most tertiary services would become dependent on the purchasing decisions of GPs. This is of considerable concern to the providers of these services, who fear that GP fundholding has led to a reduction in demand for coronary revascularization. ${ }^{2}$ Evidence from other countries of the impact that the method of funding can have on such services seems to justify their concerns. $^{34}$

The objective of this study was to provide an indication of what effect, if any, GP fundholding has had on tertiary services. An ecological study was carried out in which changes in health district rates of coronary revascularization (coronary artery bypass grafting (CABG) and percutaneous coronary angioplasty (PTCA)) were compared with the proportion of the population covered by GP fundholders.

\section{Methods and results}

The study was based on four areas of the UK - the former health regions of South East Thames, East Anglian, and North Western plus South West Scotland. Information was obtained for 36 of the 45 health districts and

Table 1 Correlation (Spearman coefficients) (95\% confidence intervals) between the proportion of district population covered by GP fundholding and the rate of coronary revascularisation (coronary artery bypass grafting (CABG) and percutaneous coronary angioplasty $(P T C A))(n=36)$.

\begin{tabular}{llrr}
\hline Fiscal year & \multicolumn{1}{l}{ CABG } & \multicolumn{1}{l}{ PTCA } & \multicolumn{1}{l}{ Both } \\
\hline $1991-92$ & $-0.07(-0.39,0.27)$ & $-0.01(-0.34,0.32)$ & $-0.07(-0.39,0.27)$ \\
$1992-93$ & $-0.24(-0.52,0.08)$ & $0.14(-0.18,0.44)$ & $0.00(-0.32,0.32)$ \\
$1993-94$ & $-0.07(-0.37,0.25)$ & $0.15(-0.18,0.44)$ & $0.06(-0.26,0.37)$ \\
$1994-95$ & $-0.26(-0.53,0.06)$ & $0.03(-0.29,0.34)$ & $-0.11(-0.41,0.22)$ \\
\hline
\end{tabular}

boards that made up these areas in 1991. Data were obtained for the four fiscal years since the start of GP fundholding (1991-92 to 1994-95). Age-sex standardised, population based NHS coronary revascularization rates were calculated for each district using data from all providers serving those populations. ${ }^{5}$ The proportion of the resident district population covered by GP fundholders was obtained from the relevant local health authority. The 1991 Carstairs index was used as an indicator of the level of socioeconomic affluence/deprivation of each district. ${ }^{6}$

In 1994-95, the proportion of the population covered by GP fundholding varied between the districts studied from about $10 \%$ to almost $80 \%$, and revascularization rates ranged from about 270 to 840 per million for CABG and from 50 to 870 per million for PTCA. As can be seen from table 1 , there was little evidence for an association between fundholding and utilisation rates. This was also true for the earlier years and for CABG and PTCA when considered separately.

Given that the rate of revascularization increased steadily over this period, ${ }^{7}$ it was possible that even though no association was apparent in the cross sectional data for each year, the adoption of fundholding could have influenced the rate of increase over time. We therefore compared the percentage increase in district CABG rates between 1991-92 and 1994-95 with the level of fundholding in 1994-95. Again, there was no association (Spearman's correlation coefficient $=0.00 ; 95 \%$ confidence intervals $-0.31,0.32$ ).

Two possible confounding factors were investigated. Firstly, there was no evidence of confounding by the rate of CABG in the initial year: districts that adopted fundholding widely had similar CABG rates in 1991-92 to those in which few GPs chose to become fundholders $(r=0.00 ;-0.30,0.29)$. Secondly, while the socioeconomic status of the district was associated with the level of fundholding in 1993-94 and 1994-95 ( $\mathrm{r}=-0.40 ;-0.64$, -0.10 ), it was not significantly associated with the CABG rate, though the confidence intervals were quite wide $(\mathrm{r}=0.27 ;-0.03,0.52)$.

\section{Comment}

The absence of any evidence that GP fundholding has affected the rate of use of coronary 
revascularization services is consistent with much of the published research on the impact of fundholding on other aspects of health care. This should serve to reassure those who have been concerned about possible adverse effects of introducing fundholding, at least for the moment. The failure to detect any effect over the first four years of fundholding, however, does not mean that this change in the organisation and funding of health care will never have any impact. We may have sought an effect too early in the implementation of fundholding, particularly as one of the regions (North Western) studied continued to maintain responsibility for purchasing revascularization services until 1993-94, and in other regions some fundholders were "bailed out" by the region if they went over-budget. In 1994-95, still only about $40 \%$ of GPs were fundholders. In other words, most coronary revascularization procedures were still being purchased by health authorities. In addition, it seems plausible that fundholders may delay changing their clinical practice and referral behaviour until the more mundane, practical aspects of managing their own budgets have become well established.

It is not possible to generalise from this study to tertiary care as a whole. In addition, most tertiary services are not yet the responsibility of GP fundholders. This, however, could change if $c$ total purchasing were to become widespread. One immediate consequence for coronary revascularization would be that the purchasing of coronary angiography would become the GP's responsibility as would the full cost of the interventions (rather than just the first $£ 6000$ as at present). There is therefore a need to continue to monitor the impact of fundholding on the use of tertiary as well as other services.

We thank Cathy Coshall for help in data collection, Colin Sanderson for advice, and the hospital and health authority staff for supplying information.

Funding: no additional funding.

Conflict of interest: None.

1 Dixon J, Glennerster $\mathrm{H}$. What do we know about fundholding in general practice? $B M \mathcal{F}$ 1995;31: 727-30.

2 Langham S, Black NA. The evolution of a public sector market for cardiac services in the UK: 1991-1994. Public Money \& Management 1995;15:31-38.

3 Wenneker M, Weissman JS, Epstein AM. The association of payer with utilisation of cardiac procedures in Massachusetts. $¥ A M A$ 1990;264:1255-60

4 Greenfield S, Nelson EC, Zubkoff $M$ et al. Variations in resource utilization among medical specialties and systems resource utilization among medical spe

5 Black NA, Langham S, Petticrew M. Why do rates of coronary revascularization vary geographically in the UK? $\mathcal{F}$ Epidemiol revascularization vary geographically in

6 Carstairs V, Lowe M. Small area analysis creating an are base for environmental monitoring and epidemiologica analysis. Community Med 1986;8: 15-17.

7 Black NA, Langham S, Petticrew $M$. Trends in the age and sex of patients undergoing coronary revascularization in the United Kingdom: 1987-1993. Br Heart $\mathcal{f} 1994 ; 72$ $317-20$ 